\title{
Mammalian target of rapamycin/p70 ribosomal S6 protein kinase signaling is altered by sevoflurane and/or surgery in aged rats
}

\author{
YONGZHE LIU, LI MA, LINBO JIAO, MINGLONG GAO, WENZHI GUO, \\ LIN CHEN, NINGLING PAN and YAQUN MA
}

Department of Anesthesiology, Beijing Military General Hospital, Beijing 100700, P.R. China

Received October 25, 2014; Accepted September 28, 2015

DOI: $10.3892 / \mathrm{mmr} .2015 .4444$

\begin{abstract}
The mammalian target of rapamycin (mTOR)/p70 ribosomal S6 protein kinase (p70S6k) pathway exerts anti-apoptotic effects that may contribute to disease pathogenesis. The memory impairment in patients with Alzheimer's disease (AD) has been suggested to be contributed to by abnormal mTOR signaling. The aim of the current study was to investigate the association between sevoflurane and/or surgery and AD through the mTOR/p70S6K signaling pathway. Sprague-Dawley rats were randomly assigned to the sevoflurane, surgery or control groups. The animals in the surgery group received a partial hepatectomy under sevoflurane anesthesia. The hippocampal levels of phosphorylated (p)-mTOR, p-p70S6K, caspase-3 and p-tau/total (t)-tau were analyzed. The Morris water maze (MWM) was used to evaluate cognitive function following treatment. The levels of p-mTOR and p-p70S6K were reduced, whereas caspase-3 levels were increased in the surgery group compared with the sevoflurane group. The p-tau/t-tau levels were increased, however, tau mRNA was unaffected by sevoflurane and/or surgery. The rats in the surgery group required a significantly longer time to locate the platform in the MWM test compared with the control and sevoflurane groups. Sevoflurane treatment and/or surgery reduced anti-apoptotic activity, and the postoperative cognitive dysfunction following surgery may be due to mTOR signaling pathway inhibition in aged rats. Increased neuronal apoptosis and tau phosphorylation are suggested to be involved in the association between anesthesia and AD occurrence.
\end{abstract}

Correspondence to: Dr Yaqun Ma, Department of Anesthesiology, Beijing Military General Hospital, 5 Nanmencang, Dongcheng, Beijing 100700, P.R. China

E-mail: lyzgao@gmail.com

Key words: mTOR signaling, tau protein, Alzheimer's disease, anesthesia, surgery

\section{Introduction}

Mammalian target of rapamycin (mTOR) is a member of the phosphoinositol kinase-related kinase family (1). mTOR is a modulator of cell growth and proliferation and regulates protein translation efficiency within cells via its downstream targets (2). Furthermore, mTOR is suggested to act primarily via the phosphorylation of eukaryotic initiation factor $4 \mathrm{E}$ binding protein (4E-BP1) and p70 ribosomal S6 protein kinase (p70S6K), which are key regulators of protein translation (3).

The mTOR pathway is suggested to contribute to long-term potentiation, long-term depression and synaptic plasticity via protein synthesis regulation (4). Rapamycin disrupts protein synthesis, which is required to stabilize short-term memory into long-term memory (5), and impairs the generation of long-term fear memories when injected into the amygdala (6). This suggests that the enhancement of neuronal mTOR activity may increase memory function. Alternatively, an increase in mTOR activity may disrupt memory processing, as demonstrated by tuberous sclerosis, which is associated with overactive mTOR. Rapamycin treatment rescued memory deficits in a transgenic mouse model of tuberous sclerosis (7), which suggests memory impairments are contributed to by abnormal mTOR signaling.

Alzheimer's disease (AD) is the most common neurodegenerative condition worldwide, in addition to being the predominant cause of dementia (8). Although no conclusive studies have identified an association between anesthesia and $\mathrm{AD}$ occurrence, the age of $\mathrm{AD}$ onset has been inversely correlated with the cumulative exposure to general anesthesia prior to the age of 50 (9). A previous study suggested that the inhaled anesthetic, sevoflurane, may increase caspase activation, in addition to inducing modest increases in $\beta$-site amyloid precursor protein-cleaving enzyme levels, which may lead to increased amyloid- $\beta$ (A $\beta$ ) levels in the mouse brain (10). Furthermore, a previous study suggested that sevoflurane accelerated the progression of amnestic mild cognitive impairment (MCI) to progressive MCI in a Chinese population (11).

Studies that have assessed human tissue have identified increased mTOR pathway activation in the affected areas of AD brains compared with non-diseased control tissue (12). mTOR activation was accompanied by reduced translational activity (13) and increased oxidative-damage to ribosomal RNA (14). However, alternative studies have demonstrated 
that $\mathrm{A} \beta$ reduced mTOR signaling (15). In addition, studies that have assessed tau are contradictory. For example, mTOR activity has been demonstrated to increase tau protein production, which suggests mTOR activation may occur upstream of tau pathology (16). Conversely, in a Drosophila melanogaster model of tauopathy, wild-type or mutant tau overexpression induced mTOR activity, neural cell cycle activation and neurodegeneration (17). $\mathrm{A} \beta_{1-42}$ has been reported to rapidly and persistently downregulate $\mathrm{mTOR} / \mathrm{p} 70 \mathrm{~S} 6 \mathrm{k}$ phosphorylation in cells, and was associated with caspase- 3 activation in a mouse model of AD (18). In addition, phosphorylated (p)-p70S6k expression was significantly reduced in the lymphocytes of patients with AD, and p-p70S6k levels were significantly correlated with Mini Mental Status Examination (MMSE) scores (18). These studies suggest that the mTOR/p70S6k pathway, which regulates cell proliferation, protein translation, apoptosis and autophagy, is altered in cellular and transgenic models of $\mathrm{AD}$, in addition to in the peripheral cells of patients, thus, these alterations may contribute to disease pathogenesis.

The current study hypothesized that mTOR/p70s6k signaling is downregulated and caspase- 3 is activated following exposure to sevoflurane and/or surgery, and that these alterations are associated with cognitive dysfunction following surgery. In the present study, the association between sevoflurane and/or surgery and AD and the role of the $\mathrm{mTOR} / \mathrm{p} 70 \mathrm{~S} 6 \mathrm{~K}$ signaling pathway was investigated in the hippocampi of aged rats.

\section{Materials and methods}

Ethics statement and animals. All experiments were approved by the Institutional Animal Ethics Committee at Beijing Military Hospital (Beijing, China) with the following approval number: 2014-01-11A. A total of 100 female Sprague-Dawley rats aged 20 months were obtained from the Laboratory Animal Center of the Academy of Military Medical Sciences (Beijing, China) and used in the experiments. The animals were group housed in a controlled environment $\left(21 \pm 2^{\circ} \mathrm{C}, 50 \%\right.$ humidity) and were maintained in a 12/12 h light/dark cycle with the lights on at $7 \mathrm{am}$. Food and water were available ad libitum throughout the experiment, with the exception of during sevoflurane and/or surgery exposure and behavioral tests.

Experimental protocol. All rats underwent baseline Morris water maze (MWM) tests, and the rats that swam slowly or remained stationary were excluded from the study. Rats $(n=100)$ were randomly assigned to the sevoflurane $(n=40)$, surgery $(n=40)$ or control $(n=20)$ groups (Fig. 1$)$. The animals in the sevoflurane group received continuous exposure to $2 \%$ sevoflurane for $2 \mathrm{~h}$. The animals in the surgery group received a partial hepatectomy under sevoflurane anesthesia and were subsequently exposed to sevoflurane anesthesia for $2 \mathrm{~h}$. A total of 15 rats in the sevoflurane and surgery groups were used for western blotting, and 15 rats were used for reverse transcription-quantitative polymerase chain reaction (RT-qPCR) analysis at 1, 3 and 7 days post-treatment, with 5 rats sacrificed by decapitation at each time point. In the control group, 5 rats were used for western blotting and 5 rats were used for RT-qPCR. The remaining 10 rats in each group underwent MWM testing at 8-12 days post-treatment.
Sevoflurane anesthesia. An anesthesia chamber $(30 \times 30 \times 15 \mathrm{~cm})$ was used to administer sevoflurane/oxygen to 5 rats at a time. Oxygen was delivered using a calibrated flow meter (LZM; Jintai Instrument Co., Ltd., Zhejiang, China) with a flow rate of $3 \mathrm{l} / \mathrm{min}$. Sevoflurane was delivered using an agent-specific vaporizer (Sevoflurane Dräger Vapor 2000; Drägerwerk AG \& Co. KGaA, Lübeck, Germany) which delivered $2 \%$ anesthetic into the chamber. The animals were maintained under the following conditions: Normothermic $\left(37-39^{\circ} \mathrm{C}\right)$, systolic blood pressure $(120-135 \mathrm{mmHg})$, diastolic blood pressure (80-100 mmHg; SurgiVet V6004; Smiths Medical PM, Inc., Norwell, MA, USA), arterial partial pressure of carbon dioxide (36-38 $\mathrm{mmHg}$ ), arterial partial pressure of oxygen $(85-88 \mathrm{mmHg})$ and hemoglobin concentration (140-120 g/1; GEM Premier 3000; Instrumentation Laboratory Co., Lexington, MA, USA) throughout the experiment. Following the initial equilibration of sevoflurane/oxygen inside the chamber, the concentrations of chamber gas composition, including sevoflurane, carbon dioxide and oxygen, were analyzed using spectrometry (FTIR-650; Guangdong Technology Co., Ltd., Tianjin, China).

Surgery. Partial hepatectomy was performed as previously described by Tanaka et al (19). The left main branch of the portal vein was dissected and ligated to the left hepatic duct and artery using a 6-0 silk (Mersilk; Johnson-Johnson Co., Ltd., Shanghai, China) ligature. When an ischemic change in the medial and left lateral lobes was identified, the left lateral lobe was ligated at the pedicle using a 3-0 silk ligature, and was subsequently removed. The median lobe at the pedicle of its left portion was ligated using a 3-0 atraumatic needle and thread, and transfixing suturing of the right portion of the pedicle was performed, following which the lobe was removed. It was necessary to make these two ligatures $5 \mathrm{~mm}$ from the inferior vena cava to avoid vessel constriction. The abdominal incision was closed using continuous sutures. The animals were subsequently exposed to sevoflurane anesthesia for $2 \mathrm{~h}$ as previously described.

Behavioral tests. Hidden platform tests were conducted in a MWM. The tests were conducted in a circular tank (DB001, Zhishu Technology Co., Ltd., Beijing, China), which was $180 \mathrm{~cm}$ in diameter and $76 \mathrm{~cm}$ deep, in a room with extra maze cues (e.g., references hung on the wall outside the pool at a height above the eye level of the rat). A $10 \mathrm{~cm}$ diameter, black round platform was placed in the center of the northeast quadrant $1.5 \mathrm{~cm}$ below the surface of the water, which was maintained at $22^{\circ} \mathrm{C}$ throughout the test duration. Animal training was conducted over a 5-day acquisition phase, during which all four quadrants were used once per day. When a rat failed to find the platform within $120 \mathrm{sec}$, it was guided to the platform by the researcher and held there for $30 \mathrm{sec}$. The training was recorded by a video camera mounted on the ceiling in conjunction with a computerized animal tracking system (Ethovision 8.0; Noldus Information Technologies, Wageningen, The Netherlands).

Western blotting. The hippocampus, which was stored in liquid nitrogen, was homogenized (F6/10-10G; FLUKO Equipment Shanghai Co., Ltd., Shanghai, China), and proteins 


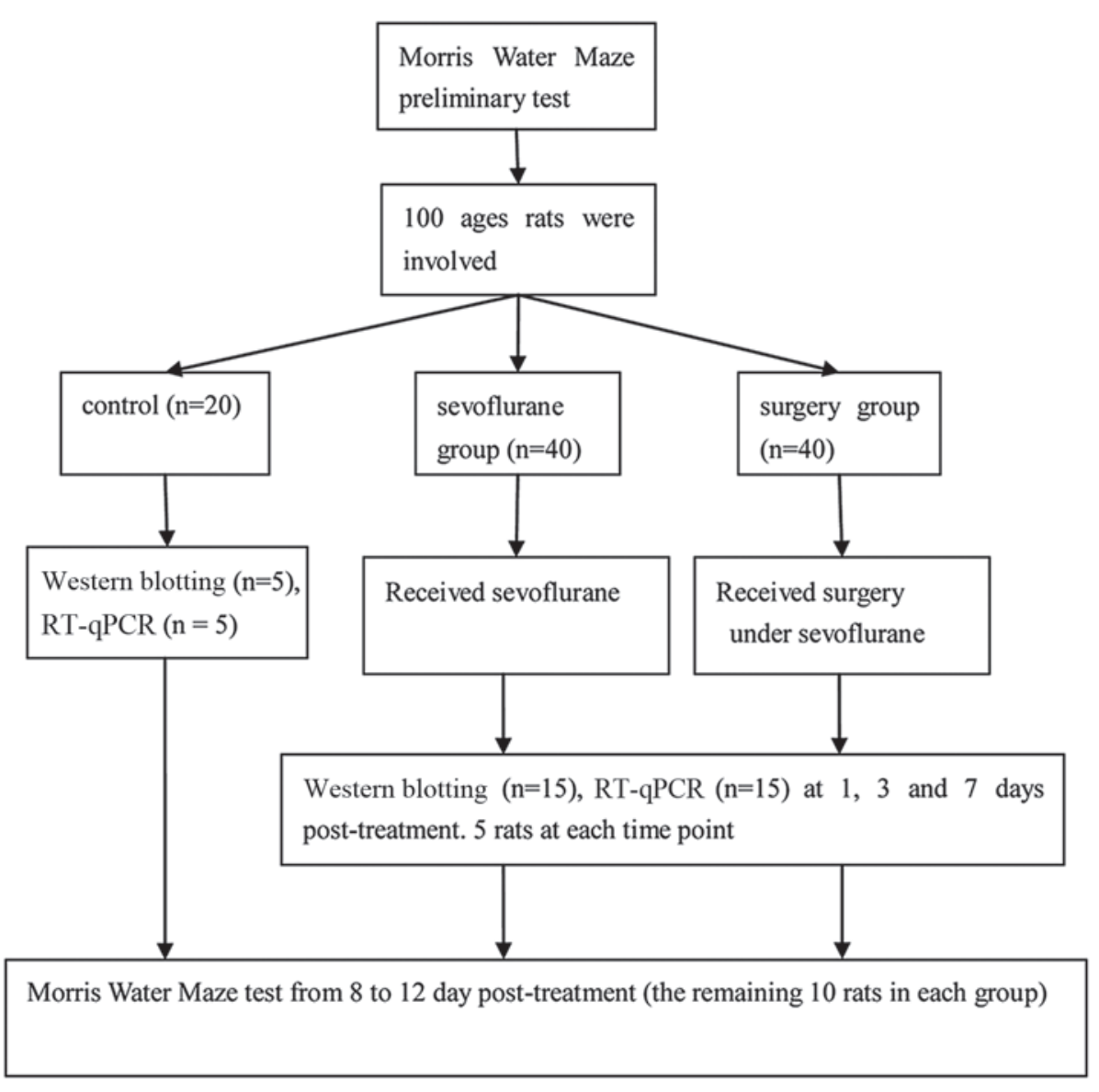

Figure 1. Schematic image of the experimental design. RT-qPCR, reverse transcription-quantitative polymerase chain reaction.

were extracted using a total protein extraction reagent kit (ProMab Biotechnologies, Inc., Richmond, CA, USA). The protein concentrations were measured using a bicinchoninic acid protein assay kit (Biyuntian Biotechnology Co., Jiangsu, China). Following denaturation at $95^{\circ} \mathrm{C}$, the proteins were separated via electrophoresis (12\% gel; Pierce Companies, Billings, MT, USA) and transferred onto nitrocellulose membranes (Pierce). The membranes were then incubated in 5\% skimmed milk (diluted in phosphate-buffered saline; Shanghai Mingrui Biotech Co., Ltd., Shanghai, China) at $37^{\circ} \mathrm{C}$ for $2 \mathrm{~h}$. The membranes were incubated with monoclonal rabbit anti-p-mTOR (ab32028; 1:1,000; Abcam, Cambridge, MA, USA), polyclonal rabbit anti-p-p70S6K (\#9205; Cell Signaling Technology, Inc., Danvers, MA, USA), monoclonal goat anti-p-tau (ab151559; Thr 231; 1:500; Abcam), polyclonal goat anti-tau (ab64193; 1:500; Abcam), and polyclonal rabbit anti-caspase-3 (1:1,000; ab2302; Abcam) antibodies at $4^{\circ} \mathrm{C}$ overnight, followed by secondary antibodies (1:40,000; ab7010; goat anti-rat $\mathrm{IgG}$; Abcam) at $37^{\circ} \mathrm{C}$ for $1.5 \mathrm{~h}$. Immunoreactive bands were detected using an enhanced chemiluminescence detection system (Cell Signaling Technology, Inc., Danvers, MA, USA). The protein levels were expressed as a band intensity ratio of the protein of interest and mouse monoclonal $\beta$-actin (1:800; SC-365062; Santa Cruz Biotechnology, Inc., Dallas, TX, USA).

RT-qPCR analysis. As previously described (20), total RNA was extracted from frozen hippocampal tissues using TRIzol reagent (Invitrogen Life Technologies, Carlsbad, CA, USA). The concentration and quantity of total RNA was measured by spectroscopy (SMA1000; Merinton Technology, Beijing, China), with an expected A260/A280 ratio of all RNA samples 1.8-2.0. The integrity of the total RNA was assessed by denaturing agarose gel electrophoresis, with the results of the $18 \mathrm{~S}$ and $28 \mathrm{~S}$ ribosomal RNA bands being clearly visible when stained with ethidium bromide. Total RNA $(2 \mu \mathrm{g})$ from each sample was reverse-transcribed in a volume of $20 \mu 1$ to produce cDNA using the first-strand cDNA synthesis kit (Invitrogen Life Technologies). The cDNA was subsequently stored at $-20^{\circ} \mathrm{C}$. RT-qPCR was performed in a volume of $25 \mu \mathrm{l}$ with 12.5 $\mu \mathrm{l}$ SYBR Green PCR Master mix (Applied Biosystems, Inc., Carlsbad, CA, USA), $1.5 \mu \mathrm{l}$ primer mix, and $1 \mu \mathrm{l}$ cDNA. All PCR reactions used the following conditions: $94^{\circ} \mathrm{C}$ for $3 \mathrm{~min}$ followed by 40 cycles at $94^{\circ} \mathrm{C}$ for $30 \mathrm{sec}$ and $72^{\circ} \mathrm{C}$ for $45 \mathrm{sec}$. The data were analyzed using the $\Delta \Delta \mathrm{Cq}$ method (21), with $\beta$-actin as the internal control. The primer sequences, the expected PCR product sizes (base pairs; bp), and the annealing temperatures were as follows: mTOR, sense 5'-TGTCGAGAG GCAACAACCTC-3' and antisense 5'-TTGTTGGCTGCA TTGTGACG-3' (298 bp, 60 C); p70S6K, sense 5'-GGATTT CTGGGGACGAGGTG-3' and antisense 5'-AGAGTTGGG CTGTCGGATTG-3' (148 bp, 58 ${ }^{\circ} \mathrm{C}$ ); tau, sense 5'-CTGAAG AAGCAGGCATCGGA-3' and antisense 5'-GGTGGTTCA CCTGATCCTGG-3' (265 bp, 59 $\left.{ }^{\circ} \mathrm{C}\right)$; caspase-3, sense 5'-CGG ACCTGTGGACCTGAAAA-3' and antisense 5'-TAA CCGGGTGCGGTAGAGTA-3' (216 bp, 60 ${ }^{\circ}$ ); $\beta$-actin, 
A p-mTOR

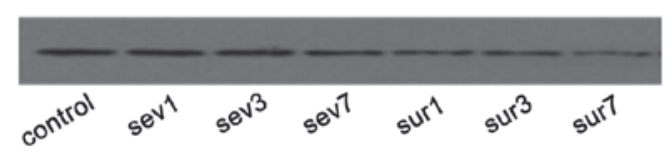

B p-p70s6K

C

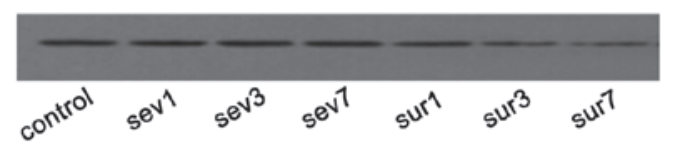

$$
\beta \text {-actin }
$$
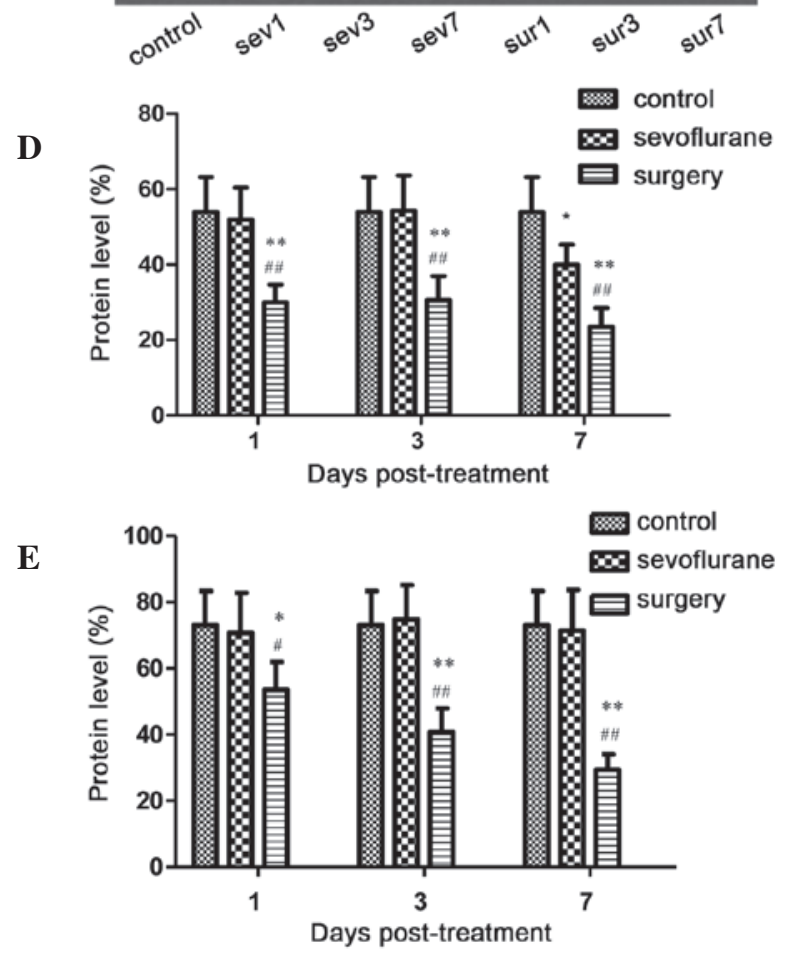

Figure 2. Western blots of p-mTOR and p-p70S6K in the hippocampus of the aged rats. Representative western blots that indicate the expression of (A) phosphorylated mTOR, (B) p70S6K, and (C) $\beta$-actin, respectively. Densitometry of the (D) p-mTOR and (E) p-p70S6K levels compared with $\beta$-actin in the hippocampus at 1,3 and 7 days after treatment. The data are presented as the mean \pm standard error of the mean, $n=5$. ${ }^{*} \mathrm{P}<0.05$ and ${ }^{* * *} \mathrm{P}<0.01$ vs. the control group. ${ }^{\#} \mathrm{P}<0.05$ and ${ }^{\# \#} \mathrm{P}<0.01$ vs. the sevoflurane group. p-mTOR, phosphorylated mammalian target of rapamycin; p-p70S6K, p70 ribosomal S6 protein kinase; sev, sevoflurane group; sur, surgery group.

sense 5'-CTCATGACCACAGTCCATGC-3' and antisense 5'-CTCATGACCACAGTCCATGC-3' (155 bp, 59 $\left.{ }^{\circ} \mathrm{C}\right)$.

Statistical analysis. Statistical analyses were performed using the SPSS 13.0 software package (SPSS Inc., Chicago, IL, USA). Two-way analysis of variance and Bonferroni post hoc test or one-way analysis of variance followed by the post hoc least significant difference test were used. $\mathrm{P}<0.05$ was considered to indicate a statistically significant difference.

\section{Results}

Sevoflurane and surgery impairs mTOR/p70S6K signaling in the hippocampus. The p-mTOR and p-p70S6K levels were
A

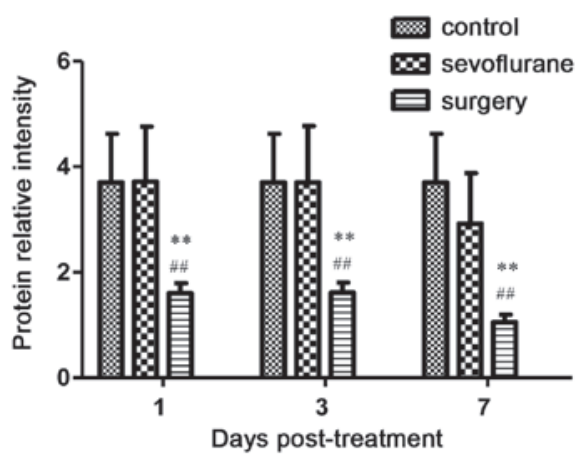

B

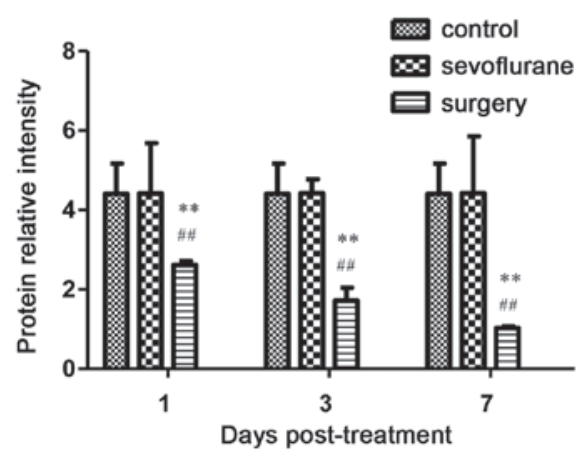

Figure 3. (A) Mammalian target of rapamycin and (B) p70 ribosomal S6 protein kinase mRNA levels determined using reverse trancription-quantitative polymerase chain reaction in aged rats. ${ }^{* *} \mathrm{P}<0.01$ vs. the control group. ${ }^{\# \#} \mathrm{P}<0.01$ vs. the sevoflurane group.

significantly reduced in the surgery group compared with the sevoflurane group on days 1,3 and 7 (Fig. 2A-E). The p-mTOR levels were significantly reduced on day 7 in the sevoflurane group and days 1, 3 and 7 in the surgery group compared with the control group $(\mathrm{P}<0.01 ;$ Fig. 2D). The p-p70S6K levels were unaltered in the sevoflurane group, however, on days 1,3 and 7, the p-p70S6K levels were significantly reduced in the surgery group compared with the control group (Fig. 2E).

The mTOR and p70S6K mRNA levels were significantly reduced in the surgery group compared with the sevoflurane group on days 1, 3 and 7. The mTOR and p70S6K mRNA levels were not significantly different in the sevoflurane group however were reduced on days 1,3 and 7 in the surgery group compared with the control group ( $\mathrm{P}<0.01$; Fig. $3 \mathrm{~A}$ and $\mathrm{B})$.

Sevoflurane and/or surgery increases caspase-3 expression in the hippocampus. The caspase-3 levels were significantly increased in the surgery group compared with the sevoflurane group on days 1, 3 and 7 (Fig. 4A-C). The caspase-3 levels were significantly increased on day 7 in the sevoflurane group, whereas the surgery group exhibited an increase at days 1 , 3 and 7 compared with the control group (Fig. 4C).

The caspase-3 mRNA levels were significantly increased in the surgery group compared with the sevoflurane group on days 1, 3 and 7, with a significant increase on day 7 in the sevoflurane group compared with the control group (Fig. 4D).

Sevoflurane and surgery, however not sevoflurane alone, increases the p-tault-tau ratio in the hippocampus. The $\mathrm{p}$-tau/t-tau ratio was significantly increased in the surgery group compared with the sevoflurane group on days 1, 3 and 7. However, in the sevoflurane group, no significant 
A

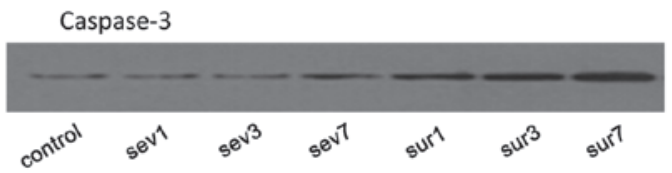

B

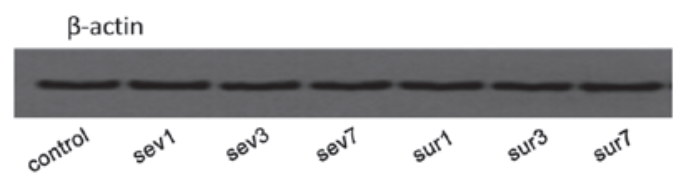

C

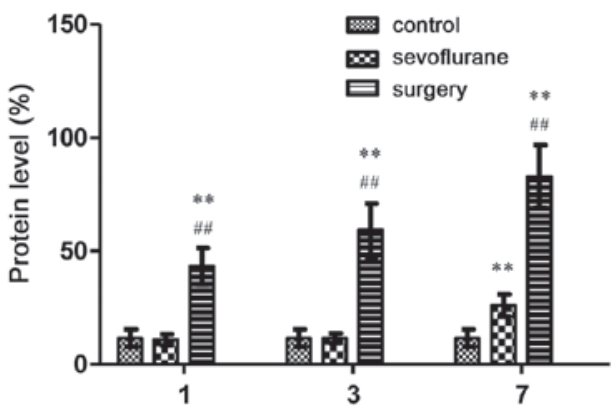

D

Days post-treatment

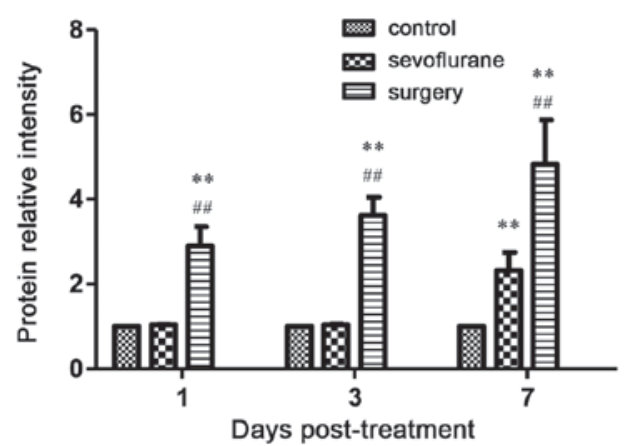

Figure 4. Representative western blots of proteins extracted from the hippocampus of aged rats and incubated with (A) anti-caspase- 3 and (B) $\beta$-actin antibodies. (C) Densitometric analysis of the levels of caspase- 3 compared with $\beta$-actin in the hippocampus on days 1, 3 and 7 in aged rats. (D) Levels of caspase- 3 mRNA measured using reverse transcription-quantitative polymerase chain reaction in aged rats. Values are presented as the mean \pm standard error, $n=5 .{ }^{* *} \mathrm{P}<0.01$ vs. the control group. ${ }^{\# \#} \mathrm{P}<0.01$ vs. the sevoflurane group. sev, sevoflurane group; sur, surgery group.

differences were observed compared with the control group (Fig. 5A-D).

RT-qPCR demonstrated that there were no significant effects of treatment or time on the caspase-3 mRNA expression (Fig. 5E). Taken together, these data suggest that the effects of sevoflurane and/or surgery on p-mTOR/p-p70S6K signaling, caspase- 3 and $\mathrm{p}$-tau/t-tau were significantly different. Of note, the effects of sevoflurane and surgery were greater than the effects of sevoflurane alone. Sevoflurane and/or surgery did not increase tau mRNA expression, which indicates the t-tau levels were not significantly different in the three groups, whereas the p-tau levels were significantly increased in the surgery group.

Sevoflurane and surgery, however not sevoflurane alone, impair cognitive function. The rats in the surgery group required a significantly longer time to locate the platform compared with the control group at all time points, and compared with the sevoflurane group on days 1,2,3 and 5. By

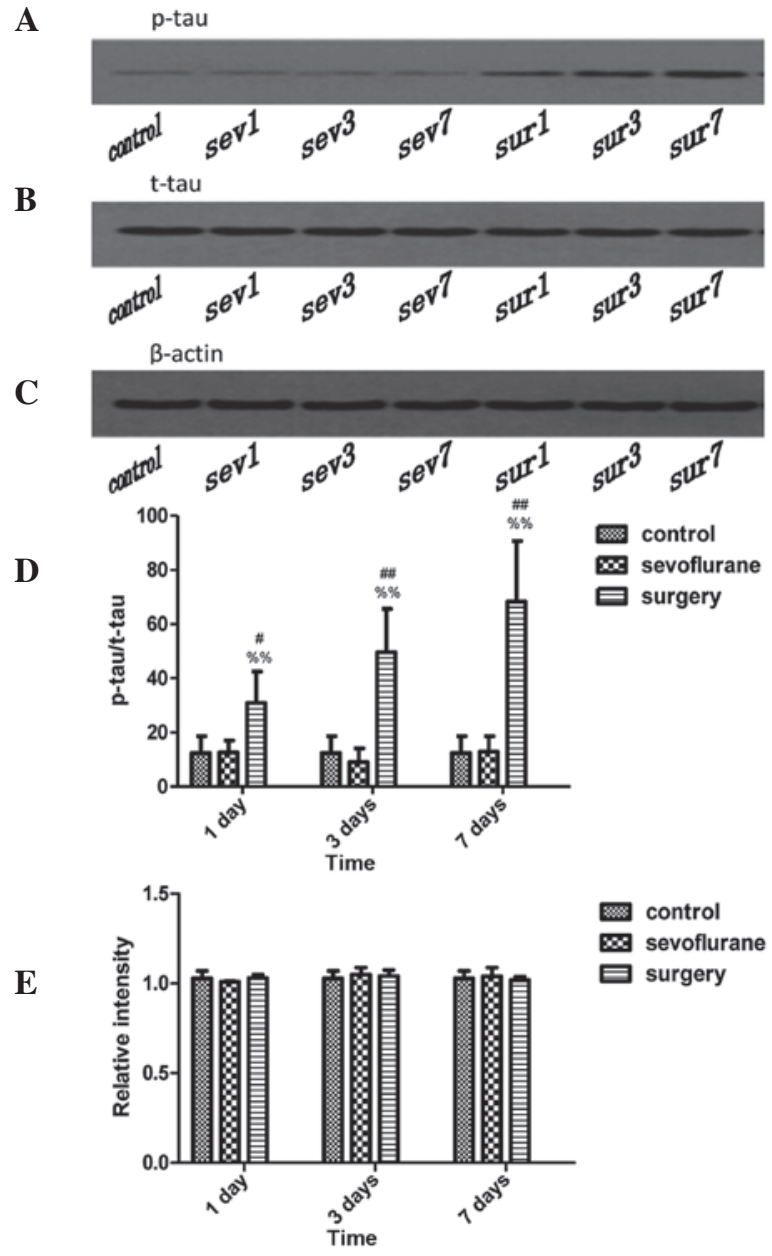

Figure 5. Representative western blotting of proteins extracted from the hippocampus of aged rats and incubated with (A) p-tau, (B) t-tau and (C) $\beta$-actin antibodies. (D) Densitometric analysis of the p-tau/t-tau ratio from blots compared with $\beta$-actin in the hippocampus on days 1,3 and 7 days in aged rats. (E) Levels of tau mRNA measured using reverse transcription-quantitative polymerase chain reaction in aged rats. Values are presented as the mean \pm standard error, $n=5$. ${ }^{*} \mathrm{P}<0.05$ and ${ }^{* *} \mathrm{P}<0.01$ vs. the control group. ${ }^{\# \#} \mathrm{P}<0.01$ vs. the sevoflurane group. $\mathrm{p}$-tau; phosphorylated tau; t-tau, total tau; sev, sevoflurane group; sur, surgery group.

contrast, there was no significant difference between the sevoflurane and control groups at any time point (Fig. 6A). The rats in the surgery group swam a longer distance compared with the control and sevoflurane groups at all time points, however, there was no significant difference between the sevoflurane and control groups (Fig. 6B).

These data suggest that the aged rats in the surgery group exhibited more substantial memory impairment compared with the sevoflurane group, and that sevoflurane alone did not lead to postoperative cognitive dysfunction.

\section{Discussion}

The current study investigated the effects of sevoflurane and/or surgery on mTOR/p70S6K signaling, caspase-3 expression and p-tau/t-tau in the hippocampus of aged rats, in addition to measuring cognitive function following treatment. These data 


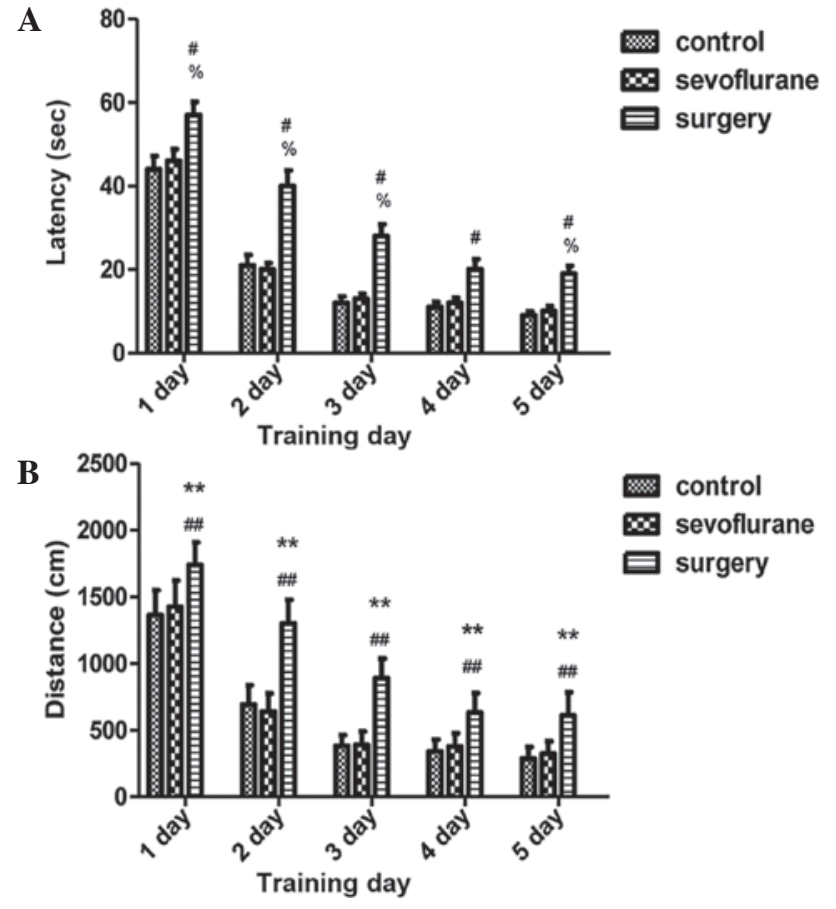

Figure 6. Rats in the surgery group exhibited greater memory decline from day 8-12 following treatment compared with those in the sevoflurane group Following treatment (days 8-12 post sevoflurane and/or surgery), the rats were trained for 5 days to find the hidden platform. (A) Latency to find the platform during the test. (B) Distance swam to the platform during the test. Values are presented as the mean \pm standard error. ${ }^{* *} \mathrm{P}<0.01$ vs. the control group. ${ }^{\#} \mathrm{P}<0.05$ and ${ }^{\# \#} \mathrm{P}<0.01$ vs. the sevoflurane group. ${ }^{\%} \mathrm{P}<0.01$ vs. the surgery group.

suggest that sevoflurane and/or surgery impairs mTOR/p70S6K signaling and increases caspase-3 expression. Sevoflurane and surgery, however not sevoflurane alone, increased p-tau/t-tau and led to postoperative cognitive dysfunction.

The aged rats in the sevoflurane group did not exhibit memory impairment in the hidden platform tests, whereas the rats in the surgery group required a significantly longer time to locate the platform at 8-12 days post-treatment. These results were consistent with those of a previous study (22). In addition to the inflammatory reaction caused by sevoflurane and the surgical procedure, the inhibition of mTOR signaling may have contributed to the cognitive decline. Previous studies have demonstrated that protein synthesis is essential for long-term synaptic plasticity (23), and synaptic protein synthesis occurs locally in dendrites and at synaptic regions (24). The inhibition of TOR signaling and its upstream activators, phosphatidylinositol 3-kinase and Akt, was demonstrated to block N-methyl-D-aspartate receptor-dependent dendritic green fluorescent protein synthesis, in addition to the dendritic synthesis of endogenous proteins involved in synaptic plasticity, including tetanic stimulation-induced calmodulin-dependent protein kinase II $\alpha(\alpha \mathrm{CaMKII})$ and microtubule-associated protein 2, in hippocampal slices (25). These studies provide evidence for the critical role of the mTOR signaling pathway in the regulation of synaptic activity-induced dendritic protein synthesis in the hippocampus. Protein synthesis is a well-established requirement for memory formation. The formation of long-term fear memory has been reported to be impaired when rapamycin is bilaterally injected into the amygdala in rats during training. Rapamycin specifically impaired memory consolidation to long-term storage, which depends on additional brain regions, including hippocampus-dependent spatial memory (26) and auditory cortex-dependent memory. The data of the current study suggest that although sevoflurane and/or surgery impaired mTOR signaling in the hippocampus, cognitive function declined only in aged rats in the sevoflurane and surgery group. It is possible that sevoflurane alone has a weaker effect on mTOR signaling and cognitive function compared with the combination of sevoflurane and surgery.

mTOR phosphorylates p70S6k at Thr 389, and a previous study demonstrated that $A \beta_{1-42}$ produced a rapid and persistent downregulation of $\mathrm{mTOR} / \mathrm{p} 70 \mathrm{~S} 6 \mathrm{k}$ phosphorylation in murine neuroblastoma cells, which was associated with caspase-3 activation (27). p-p70S6k expression has been observed to be significantly reduced in the lymphocytes of patients with AD, with p-p70S6k levels significantly correlated with the MMSE scores (18). mTOR inhibition via rapamycin led to an increase in $A \beta$-induced cell death in cultured cells (27). This observation suggests that $\mathrm{mTOR} / \mathrm{p} 70 \mathrm{~S} 6 \mathrm{~K}$ activity is beneficial for cell survival, and that mTOR/p70S6k pathway inhibition may contribute to the degenerative process in the brains of patients with AD. The data of the current study are consistent with a previous study (18). In the current study. sevoflurane and/or surgery inhibited mTOR/p70S6K signaling and reduced caspase-3 levels in the hippocampus. However, previous studies have suggested that neurofibrillary tangle formation and tau phosphorylation are significantly associated with increased mTOR phosphorylation, in addition to with its targets, p70S6K and 4E-BP1 $(12,28)$. Previously, the levels of p-p70S6K at the Thr 421/Ser 424 and Thr 389 sites were observed to correspond with increased tau phosphorylation at Ser 262 (29). Regression analyses have demonstrated that $\mathrm{t}$-tau and p-tau levels were dependent on p-p70S6K, however not at the site typically phosphorylated by mTOR (28), which suggests mTOR may have both positive and negative effects. In the current study, the mTOR-mediated p-p70S6K levels were measured, which demonstrated that sevoflurane and surgery increased p-tau/-tau in the hippocampus, whereas sevoflurane alone did not increase the ratio. Further studies are required to investigate additional pathways that may induce tau phosphorylation.

A previous study demonstrated that exposure to clinically relevant doses of isoflurane increased tau phosphorylation and insoluble and aggregated forms of tau, and induced the detachment of tau from microtubules (30). The insoluble tau levels were increased one week following anesthesia, which suggests that anesthesia precipitates brain alterations which may promote the subsequent development of tauopathy (30). Among the kinases that are able to phosphorylate tau, glycogen synthase kinase-3 $\beta$, cyclin-dependent kinase-5, mitogen-activated protein kinase/extracellular signal-regulated kinase, p38 and c-Jun N-terminal kinase are considered the key physiological and pathological tau kinases (31-33). Furthermore, CaMKII has been suggested to serve a major role in the regulation of tau phosphorylation at the epitopes that modulate tau binding to microtubules $(34,35)$.

Anesthesia-induced hypothermia leads to overt tau hyperphosphorylation in normal mouse brains, however, the duration of anesthesia or the types of anesthetics were not observed to 
alter the pattern of hypothermia-induced kinase activity. One week following a $4 \mathrm{~h}$ exposure to isoflurane, all kinase profiles were identical compared with the control-treated animals (30). Alternatively, studies have suggested that tau hyperphosphorylation is associated with abnormal glucose metabolism due to hypothermia (36). However, in the current study, the body temperature of the rats was maintained at $\sim 37^{\circ} \mathrm{C}$, which thus eliminates the potential effect of body temperature on tau phosphorylation.

In the current study, the effects of sevoflurane and surgery on postoperative cognitive dysfunction, mTOR signaling and caspase-3 were observed to be greater than that of sevoflurane anesthesia alone. A previous study has additionally demonstrated that 18 month-old mice that underwent abdominal surgery with local anesthesia alone exhibited cognitive impairment (37). The surgery alone induced neuroinflammation which may result in cognitive dysfunction through the inhibition of synaptic function and neurogenesis, and may contribute to post-operative cognitive dysfunction $(38,39)$. In addition, the stress and pain associated with surgery may contribute to cognitive dysfunction $(40,41)$. Therefore, the alterations in mTOR signaling and caspase-3 resulting from anesthesia and surgery in the current study may be associated with this.

The current study has several limitations. Due to the use of multiple techniques, the sample size of each group was small, therefore, future studies are required to verify these data using a larger sample of animals. The present study did not investigate the effect of restoring $\mathrm{mTOR} / \mathrm{p} 70 \mathrm{~S} 6 \mathrm{~K}$ signaling on the alterations in caspase- 3 or post-operative cognitive function. Therefore future studies may undertake this approach to further reinforce the current evidence. Furthermore, future studies are required to investigate the association between the mTOR signaling pathway and post-operative cognitive dysfunction.

In conclusion, compared with the sevoflurane group, the levels of hippocampal p-mTOR and p-p70S6K were reduced in the surgery group, whereas the caspase- 3 levels were increased. Surgery increased the ratio of $\mathrm{p}$-tau/t-tau in the hippocampus of aged rats. The postoperative cognitive dysfunction following surgery is, in part, a result of mTOR signaling pathway inhibition in aged rats. Overall, the present study suggests that increased neuronal apoptosis and tau phosphorylation may be involved in the association between anesthesia and the occurrence of AD. It is thus suggested taht sevoflurane anesthesia should not be the first choice in patients with $\mathrm{AD}$ or high-risk groups, however further investigation is required to verify this.

\section{Acknowledgements}

The current study was supported by the Department of Anesthesiology, Beijing Military General Hospital (Beijing, China).

\section{References}

1. Sarbassov DD, Guertin DA, Ali SM and Sabatini DM: Phosphorylation and regulation of Akt/PKB by the rictor-mTOR complex. Science 307: 1098-1101, 2005.

2. Schmelzle T and Hall MN: TOR, a central controller of cell growth. Cell 103: 253-262, 2000.
3. Burnett PE, Barrow RK, Cohen NA, Snyder SH and Sabatini DM: RAFT1 phosphorylation of the translational regulators p70 S6 kinase and 4E-BP1. Proc Natl Acad Sci USA 95: 1432-1437, 1998.

4. Tang SJ, Reis G, Kang H, Gingras AC, Sonenberg N and Schuman EM: A rapamycin-sensitive signaling pathway contributes to long-term synaptic plasticity in the hippocampus. Proc Natl Acad Sci USA 99: 467-472, 2002.

5. Abel T and Lattal KM: Molecular mechanisms of memory acquisition, consolidation and retrieval. Curr Opin Neurobiol 11: 180-187, 2001.

6. Parsons RG, Gafford GM and Helmstetter FJ: Translational control via the mammalian target of rapamycin pathway is critical for the formation and stability of long-term fear memory in amygdala neurons. J Neurosci 26: 12977-12983, 2006.

7. Ehninger D, Han S, Shilyansky C, Zhou Y,Li W, Kwiatkowski DJ, Ramesh V and Silva AJ: Reversal of learning deficits in a Tsc2+/mouse model of tuberous sclerosis. Nat Med 14: 843-848, 2008.

8. Masters CL, Cappai R, Barnham KJ and Villemagne VL: Molecular mechanisms for Alzheimer's disease: Implications for neuroimaging and therapeutics. J Neurochem 97: 1700-1725, 2006.

9. Bohnen N, Warner MA, Kokmen E and Kurland LT: Early and midlife exposure to anesthesia and age of onset of Alzheimer's disease. Int J Neurosci 77: 181-185, 1994.

10. Dong Y, Zhang G, Zhang B, Moir RD, Xia W, Marcantonio ER, Culley DJ, Crosby G, Tanzi RE and Xie Z: The common inhalational anesthetic sevoflurane induces apoptosis and increases beta-amyloid protein levels. Arch Neurol 66: 620-631, 2009.

11. Liu Y, Pan N, Ma Y, Zhang S, Guo W, Li H, Zhou J, Liu G and Gao M: Inhaled sevoflurane may promote progression of amnestic mild cognitive impairment: A prospective, randomized parallel-group study. Am J Med Sci 345: 355-360, 2013.

12. Li X, Alafuzoff I, Soininen H, Winblad B and Pei JJ: Levels of mTOR and its downstream targets 4E-BP1, eEF2, and eEF2 kinase in relationships with tau in Alzheimer's disease brain. FEBS J 272: 4211-4220, 2005.

13. Langstrom NS, Anderson JP, Lindroos HG, Winblad B and Wallace WC: Alzheimer's disease-associated reduction of polysomal mRNA translation. Brain Res Mol Brain Res 5: 259-269, 1989.

14. Ding Q, Markesbery WR, Chen Q, Li F and Keller JN: Ribosome dysfunction is an early event in Alzheimer's disease. J Neurosci 25: 9171-9175, 2005

15. Swiech L, Perycz M, Malik A and Jaworski J: Role of mTOR in physiology and pathology of the nervous system. Biochim Biophys Acta 1784: 116-132, 2008.

16. Meske V, Albert F and Ohm TG: Coupling of mammalian target of rapamycin with phosphoinositide 3-kinase signaling pathway regulates protein phosphatase $2 \mathrm{~A}$ - and glycogen synthase kinase-3 -dependent phosphorylation of Tau. J Biol Chem 283: 100-109, 2008.

17. Khurana V, Lu Y, Steinhilb ML, Oldham S, Shulman JM and Feany MB: TOR-mediated cell-cycle activation causes neurodegeneration in a Drosophila tauopathy model. Curr Biol 16: 230-241, 2006

18. Lafay-Chebassier C, Paccalin M, Page G, Barc-Pain S, Perault-Pochat MC, Gil R, Pradier L and Hugon J: mTOR/p70S6k signalling alteration by Abeta exposure as well as in APP-PS1 transgenic models and in patients with Alzheimer's disease. J Neurochem 94: 215-225, 2005.

19. Tanaka H, Hashizume K, Enosawa S and Suzuki S: Successful transplantation of a $20 \%$ partial liver graft in rats: A technical innovation. J Surg Res 110: 409-412, 2003.

20. Liu Y, Gao M, Ma L, Zhang L and Pan N: Sevoflurane alters the expression of receptors and enzymes involved in $\mathrm{A} \beta$ clearance in rats. Acta Anaesthesiol Scand 57: 903-910, 2013.

21. Zhou W, Wang G, Zhao X, Xiong F, Zhou S, Peng J, Cheng Y, $\mathrm{Xu} \mathrm{S}$ and Xu X: A multiplex qPCR gene dosage assay for rapid genotyping and large-scale population screening for deletional $\alpha$-thalassemia. J Mol Diagn 15: 642-651, 2013.

22. Li M, Yong-Zhe L, Ya-Qun M, Sheng-Suo Z, Li-Tao Z and Ning-Ling P: Ulinastatin alleviates neuroinflammation but fails to improve cognitive function in aged rats following partial hepatectomy. Neurochem Res 38: 1070-1077, 2013.

23. Kandel ER: The molecular biology of memory storage: A dialogue between genes and synapses. Science 294: 1030-1038, 2001.

24. Steward O and Schuman EM: Protein synthesis at synaptic sites on dendrites. Annu Rev Neurosci 24: 299-325, 2001. 
25. Gong R, Park CS, Abbassi NR and Tang SJ: Roles of glutamate receptors and the mammalian target of rapamycin (mTOR) signaling pathway in activity-dependent dendritic protein synthesis in hippocampal neurons. J Biol Chem 281: 18802-18815, 2006.

26. Dash PK, Orsi SA and Moore AN: Spatial memory formation and memory-enhancing effect of glucose involves activation of the tuberous sclerosis complex-Mammalian target of rapamycin pathway. J Neurosci 26: 8048-8056, 2006.

27. Lafay-Chebassier C, Pérault-Pochat MC, Page G, Rioux Bilan A, Damjanac M, Pain S, Houeto JL, Gil R and Hugon J: The immunosuppressant rapamycin exacerbates neurotoxicity of Abeta peptide. J Neurosci Res 84: 1323-1334, 2006.

28. An WL, Cowburn RF, Li L, Braak H, Alafuzoff I, Iqbal K, Iqbal IG, Winblad B and Pei JJ: Up-regulation of phosphorylated/activated p70 S6 kinase and its relationship to neurofibrillary pathology in Alzheimer's disease. Am J Pathol 163: 591-607, 2003.

29. Zhou XW, Tanila H and Pei JJ: Parallel increase in p70 kinase activation and tau phosphorylation (S262) with Abeta overproduction. FEBS Lett 582: 159-164, 2008.

30. Planel E, Bretteville A, Liu L, Virag L, Du AL, Yu WH, Dickson DW, Whittington RA and Duff KE: Acceleration and persistence of neurofibrillary pathology in a mouse model of tauopathy following anesthesia. FASEB J 23: 2595-2604, 2009.

31. Maccioni RB, Otth C, Concha II and Muñoz JP: The protein kinase Cdk5. Structural aspects, roles in neurogenesis and involvement in Alzheimer's pathology. Eur J Biochem 268: 1518-1527, 2001

32. Zhu X, Lee HG, Raina AK, Perry G and Smith MA: The role of mitogen-activated protein kinase pathways in Alzheimer's disease. Neurosignals 11: 270-281, 2002.

33. Planel E, Sun X and Takashima A: Role of GSK-3 beta in Alzheimer's disease pathology. Drug Dev Res 56: 491-510, 2002.
34. Litersky JM, Johnson GV, Jakes R, Goedert M, Lee M and SeubertP: Tau protein is phosphorylated by cyclic AMP-dependent protein kinase and calcium/calmodulin-dependent protein kinase II within its microtubule-binding domains at Ser-262 and Ser-356. Biochem J 316: 655-660, 1996.

35. Sironi JJ, Yen SH, Gondal JA, Wu Q, Grundke-Iqbal I and IqbalK: Ser-262 in human recombinant tau protein is a markedly more favorable site for phosphorylation by CaMKII than PKA or PhK. FEBS Lett 436: 471-475, 1998.

36. Planel E, Miyasaka T, Launey T, Chui DH, Tanemura K, Sato S, Murayama O, Ishiguro K, Tatebayashi Y and Takashima A: Alterations in glucose metabolism induce hypothermia leading to tau hyperphosphorylation through differential inhibition of kinase and phosphatase activities: implications for Alzheimer's disease. J Neurosci 24: 2401-2411, 2004.

37. Xu Z, Dong Y, Wang H, Culley DJ, Marcantonio ER, Crosby G, Tanzi RE, Zhang Y and Xie Z: Age-dependent postoperative cognitive impairment and Alzheimer-related neuropathology in mice. Sci Rep 4: 3766, 2014.

38. Terrando N, Monaco C, Ma D, Foxwell BM, Feldmann M and Maze M: Tumor necrosis factor-alpha triggers a cytokine cascade yielding postoperative cognitive decline. Proc Natl Acad Sci USA 107: 20518-20522, 2010.

39. Lyman M, Lloyd DG, Ji X, Vizcaychipi MP and Ma D Neuroinflammation: The role and consequences. Neurosci Res 79: 1-12, 2014.

40. Marcos B, Aisa B and Ramírez MJ: Functional interaction between 5-HT(6) receptors and hypothalamic-pituitary-adrenal axis: Cognitive implications. Neuropharmacology 54: 708-714, 2008.

41. Hu Y, Yang J, Hu Y, Wang Y and Li W: Amitriptyline rather than lornoxicam ameliorates neuropathic pain-induced deficits in abilities of spatial learning and memory. Eur J Anaesthesiol 27: 162-168, 2010. 